NEUROLOGICAL DISORDERS

\section{For emotion, size matters}



Individuals with Williams syndrome, a genetic developmental condition that causes deficits in visuospatial processing but enhanced emotionality and face processing, have decreases and increases in relative cortical volume that parallel these abnormalities.

In a study published in The Journal of Neuroscience, Reiss and colleagues used a combination of volumetric analysis and voxel-based morphometry to compare the brains of people with Williams syndrome and those of control subjects. Williams syndrome causes various cognitive and behavioural abnormalities, including mild to moderate intellectual deficits, but visuospatial processing is disproportionately affected. The authors hypothesized that the parts of the cortex that make up the 'dorsal stream', which is responsible for visuospatial processing, might be abnormally small in affected individuals. They also looked for changes in parts of the brain that are involved in emotion and face processing, as people with the syndrome show increased emotional behaviour and are relatively good at face processing.

Overall, the study found that patients with Williams syndrome had an $11 \%$ reduction in cerebral volume. However, the thalamus, occipital cortex and other areas that are involved in visuospatial processing had greater decreases in grey matter volume, consistent with the pronounced deficits seen in these individuals. When the authors looked at areas involved with emotional processing and behaviour including the amygdala, orbital and medial prefrontal cortex and anterior cingulate cortex - they found that these areas were relatively larger than normal.

\title{
Different shades of colour blindness
}

Despite the prevalence of colour blindness among men, and a good understanding of the genetics that underlies the condition, it has been unclear how the genetic abnormalities affect the cone receptors of the retina. Now, Carroll and colleagues use adaptive optics to show that there are two distinct phenotypes, caused by different types of genetic abnormality.

In individuals with $\mathrm{X}$-linked red-green colour blindness, the function of either the long-wavelength $(\mathrm{L})$ or middle-wavelength (M) photoreceptor pigment is lost. A central question in colour blindness research is whether these individuals have a normal complement of cone photoreceptors - but with the absent photopigment replaced by the remaining one - or a loss of the cones that would normally express the missing pigment.

Carroll et al. investigated this question in two red-green colour-blind men. Genetic analysis showed that in one individual (MM) the L-pigment gene on the $\mathrm{X}$ chromosome had been replaced by a second copy of the M-pigment gene. In the other (NC), by contrast, the M-pigment gene contained a mutation that caused it to encode a non-functional pigment.

The authors used a combination of two techniques - adaptive optics and retinal densitometry - to visualize the cone photoreceptors in the retinas of $\mathrm{MM}$ and $\mathrm{NC}$, and to compare them with those in a normal trichromatic retina. In MM, they found a normal density of cones in the retina, which supports the idea that those cones that should contain the L pigment are still there, but contain the M pigment instead. However, NC's retina showed dark patches that appeared to correspond to missing $M$ cones, scattered among a normal arrangement of $\mathrm{L}$ and $\mathrm{S}$ cones. In this case, it seems that the $\mathrm{M}$ cones had died, or at least become so abnormal that they did not reflect light as they should, and so could not be visualized. Surprisingly, the loss of about one-third of the cones in NC's retina did not significantly impair other aspects of his vision.
Until this study, the 'replacement' theory of colour blindness - the idea that dichromats had a normal number of cones, but only two instead of three pigments had been more popular than the idea that cones were lost. These new findings show that both theories are apparently correct, depending on the genetic cause of the condition. They also demonstrate the usefulness of the high-resolution visualization of the retina that is allowed by adaptive optics - a technique that could prove invaluable for both research into and earlier diagnosis of retinal abnormalities.

Rachel Jones

(9) References and links ORIGINAL RESEARCH PAPER Carroll, J. et al. Functional photoreceptor loss revealed with adaptive optics: an alternate cause of color blindness. Proc. Natl Acad. Sci. USA 101, 8461-8466 (2004)

FURTHER READING Dacey, D. M. \& Packer, O. S. Colour coding in the primate retina: diverse cell types and conespecific circuitry. Curr. Opin. Neurobiol. 13, 421-427 (2003) WEB SITE

Williams laboratory: http://www.cvs.rochester.edu/people/ d_williams/d_williams.html 